EPJ Web of Conferences 92,02054 (2015)

DOI: $10.1051 /$ epjconf/ 20159202054

(C) Owned by the authors, published by EDP Sciences, 2015

\title{
Prediction of surface tension of binary mixtures with the parachor method
}

\author{
Tomáš Němec ${ }^{1, \mathrm{a}}$ \\ ${ }^{1}$ Institute of Thermomechanics ASCR, v.v.i., Dolejškova 5, 18200 Praha 8, Czech Republic
}

\begin{abstract}
The parachor method for the estimation of the surface tension of binary mixtures is modified by considering temperature-dependent values of the parachor parameters. The temperature dependence is calculated by a least-squares fit of pure-solvent surface tension data to the binary parachor equation utilizing the Peng-Robinson equation of state for the calculation of equilibrium densities. A very good agreement between experimental binary surface tension data and the predictions of the modified parachor method are found for the case of the mixtures of carbon dioxide and butane, benzene, and cyclohexane, respectively. The surface tension is also predicted for three refrigerant mixtures, i.e. propane, isobutane, and chlorodifluoromethane, with carbon dioxide.
\end{abstract}

\section{Introduction}

The parachor method [1] relates the surface tension of liquids to the densities of the liquid and the vapor phase. In the simplest case of a pure substance, the surface tension $\sigma[\mathrm{N} / \mathrm{m}]$ is evaluated as $\sigma^{1 / 4}=P\left(\varrho_{l}-\varrho_{v}\right)[2,3]$, where $\varrho_{l}$ $\left[\mathrm{mol} / \mathrm{m}^{3}\right]$ is the density of the liquid phase and $\varrho_{v}\left[\mathrm{~mol} / \mathrm{m}^{3}\right]$ is the density of the vapor phase. The relation involves a substance-specific constant $P$ called the parachor. Its value was estimated for a wide range of substances from experimental surface tension data $[4,5]$. Also, a correlation between the parachor and the critical volume was identified [6].

More advanced methods of the surface tension prediction are based on the gradient theory coupled with various equations of state, e.g. the Peng-Robinson EOS [7], PCSAFT EOS [8], or PCP-SAFT EOS [9]. However, the parachor method presents a simple alternative, with a straightforward implementation and a sufficient precision for engineering applications.

In the case of multicomponent mixtures of $N$ components the parachor relation takes the form

$$
\sigma=\left(\sum_{i=1}^{N} P_{i}\left(\varrho_{l} x_{i}-\varrho_{v} y_{i}\right)\right)^{n},
$$

where $x_{i}$ is the mole fraction of the liquid component $i$, and $y_{i}$ is the mole fraction of the vapor component $i$. Several binary systems were already investigated with the parachor method $[10,11]$, and a good agreement with available experimental surface tension data [12,13] was observed.

The exponent $n$ in Eq. (1) was often discussed in the literature, and, instead of the original value of 4 , values of $3.57,3.66$, or 3.88 were found more appropriate in some cases. Higher exponents seem more suitable for higher densities (intermediate temperatures), while lower exponents

a e-mail:nemec@it.cas.cz work better for lower densities (close to the critical point); see $[5,14]$ for a more detailed discussion.

Also, the constant nature of the parachor was put to question. Zhelezny et al.[6] investigated the temperature dependence of the parachor, and they found a considerable increase, by tens of percent, in the parachor value in the limit of the critical point.

In this work, the parachor method will be applied to the prediction of the surface tension of binary mixtures of carbon dioxide. The goal is to investigate the possible temperature-dependence of the parachor parameters, i.e. the parachors $P_{i}$ and the exponent $n$ in Eq. (1), or, in other words, to assess the improvement in predictive capability of the parachor method by allowing the parachor parameters to vary with temperature. In section 2 , an overview of the method is given. In section 3.1, the surface tension predicted with constant parachor parameters is compared to the available experimental data for the binary mixtures of carbon dioxide and n-butane, benzene, and cyclohexane, respectively. In section 3.2, the derivation of the temperature-dependent parachor parameters is given, and the predictions of the parachor method with temperaturedependent parameters are presented. In section 3.3, the surface tension of the refrigerant mixtures of carbon dioxide and propane, isobutane, and chlorodifluoromethane (R22), respectively, is predicted. The surface tension of these refrigerant mixtures is valuable for nucleation studies [15], since no experimental data are available so far. Finally, in section 3.4, the scaling behavior of the temperaturedependent parachor is studied, focusing on the correlation between the parachor and the critical volume of a given substance [6].

\section{Parachor method}

In order to estimate the surface tension of a binary mixture according to Eq. (1), the densities of the vapor phase $\varrho_{v}$ and 
of the liquid phase $\varrho_{l}$ are required at the vapor-liquid equilibrium (VLE). For the binary mixtures considered in this work, except R22, the available experimental VLE data from the literature are accurately represented by the predictive Peng-Robinson equation of state (PPR78 EOS) of Vitu et al. [16]. PPR78 features a temperature-dependent interaction parameter $k_{i j}$. In order to evaluate the PPR78 equation, the values of $k_{i j}$ given in Vitu's work were fitted to a polynomial function of temperature. For the case of R22, the original Peng-Robinson EOS was used with the interaction parameter $k_{i j}=0.009$, as predicted by Vrabec $e t$ al. [17].

The calculation of the surface tension with the parachor method proceeds as follows. The surface tension is being evaluated as a function of the temperature $T[\mathrm{~K}]$ and the composition of the liquid phase, given by the carbon dioxide mole fraction $x_{2}$. The respective EOS is used to evaluate the corresponding equilibrium composition of the vapor phase, $y_{2}$, and the vapor pressure $p[\mathrm{~Pa}]$, by means of a bubble-point calculation (see e.g. Smith and Van Ness [18], p. 490). Having the equilibrium compositions $x_{2}, y_{2}$ at hand, the equilibrium vapor and liquid densities $\varrho_{v}$ and $\varrho_{l}$ are obtained by solving the cubic EOS at $p, T$, and $x_{2}$, or $y_{2}$, respectively. This procedure results in the evaluation of $y_{i}$, $\varrho_{v}$, and $\varrho_{l}$ for use in Eq. (1) at a given $T$, and $x_{2}$.

Now, to evaluate Eq. (1), the parachor parameters $P_{1}$, $P_{2}, n$ remain to be specified. Either the value reported in the literature can be used, or the parameters can be fitted to available experimental data with Eq. (1). Both approaches will be discussed further, focusing of the possibility of the temperature dependence of the parachor parameters.

\section{Results and discussion}

Two different cases will be discussed in order to study the possibility of a temperature dependence of the parachor parameters.

First, constant values of $P_{1}, P_{2}$, and $n$ will be assumed. This is a standard approach, applied for example by Gasem et al. [10], who fitted the three parameters to experimental VLE data (i.e. surface tension, liquid and vapor densities) of binary mixtures. The estimated surface tension based on the parachors of Gasem et al. is discussed in section 3.1.

Second, temperature-dependent values of $P_{1}$ and $n$ will be considered. The parachor of carbon dioxide is taken constant $P_{2}=79.2$ [10] in this case, because all the mixtures investigated in this work are studied above the critical point of the pure carbon dioxide, and therefore the dependence on its reduced temperature is irrelevant. The predictions of the surface tension by the parachor method in case the model parameters $P_{1}$ and $n$ are allowed to vary with temperature are discussed in section 3.2.

\subsection{Constant parachors}

In the work of Gasem et al. [10] the parachors of several substances were calculated from the experimental data for the VLE densities and the surface tension of binary mixtures. A least-squares optimizing procedure was applied to
Table 1. Parachors by Gasem et al. [10] corresponding to the exponent $n=3.57$ of Eq. (1).

\begin{tabular}{ll}
\hline Compound & Parachor \\
\hline carbon dioxide & 79.2 \\
n-butane & 199.7 \\
benzene & 200.4 \\
cyclohexane & 234.9 \\
\hline
\end{tabular}

fit the parameters $P_{1}, P_{2}$, and $n$ to the available experimental data for mixtures of carbon dioxide and n-butane, benzene, cyclohexane. The value of the exponent fitted by Gasem et al. is $n=3.57$, and their parachor values are given in table 1.

The surface tension of the three above mentioned binary mixtures calculated according to Eq. (1) with the parachor parameters from table 1 are shown in figure 1 . In all three cases the experimental binary surface tension data (circles) are reproduced satisfactorily. However, large discrepancies can be observed in the surface tensions of pure solvents (squares), especially in the cases of benzene and cyclohexane. Moreover, no definite rule can be spotted in the nature of this discrepancy; the surface tension is both overestimated (butane at low temperatures), but also underestimated (other cases). For benzene, the measured surface tension is $35 \%$ higher than the surface tension predicted by the parachor method. This presents a strong argument for parachors and/or exponent being dependent on the thermodynamic state of the system rather than being universal constants.

\subsection{Temperature dependent parachor and exponent}

The possibility of the temperature dependence of the parachor parameters will be investigated in the following two cases:

case 1 The value of $P_{1}$ is allowed to vary with temperature, while the exponent is assumed constant, $n=4$.

case 2 Both parameters $P_{1}$ and $n$ are considered temperaturedependent.

As already noted, the parachor of the carbon dioxide is taken constant, $P_{2}=79.2$. Actually, the precise value of $P_{2}$ does not have a significant influence on the results. Only in case a considerably different value of $P_{2}$ would be used, e.g. multiplied by a factor of 2 , the convexity of the resulting functional dependence $\sigma\left(x_{2}\right)$ would be slightly changed.

In both the above specified cases, the parachor parameters are calculated from the available experimental surface tension data of the pure solvents only. No binary experimental surface tension data are involved in the calculation. The two parameters $P_{1}$ and $n$ of Eq. (1) are fitted to the surface tension data with a least-squares optimization procedure implemented in Python ${ }^{1}$.

Figure 2 shows the results of the optimization procedure for the case 1 . The parachors of the solvents are fitted

\footnotetext{
1 scipy.optimize.minpack.leastsq
} 

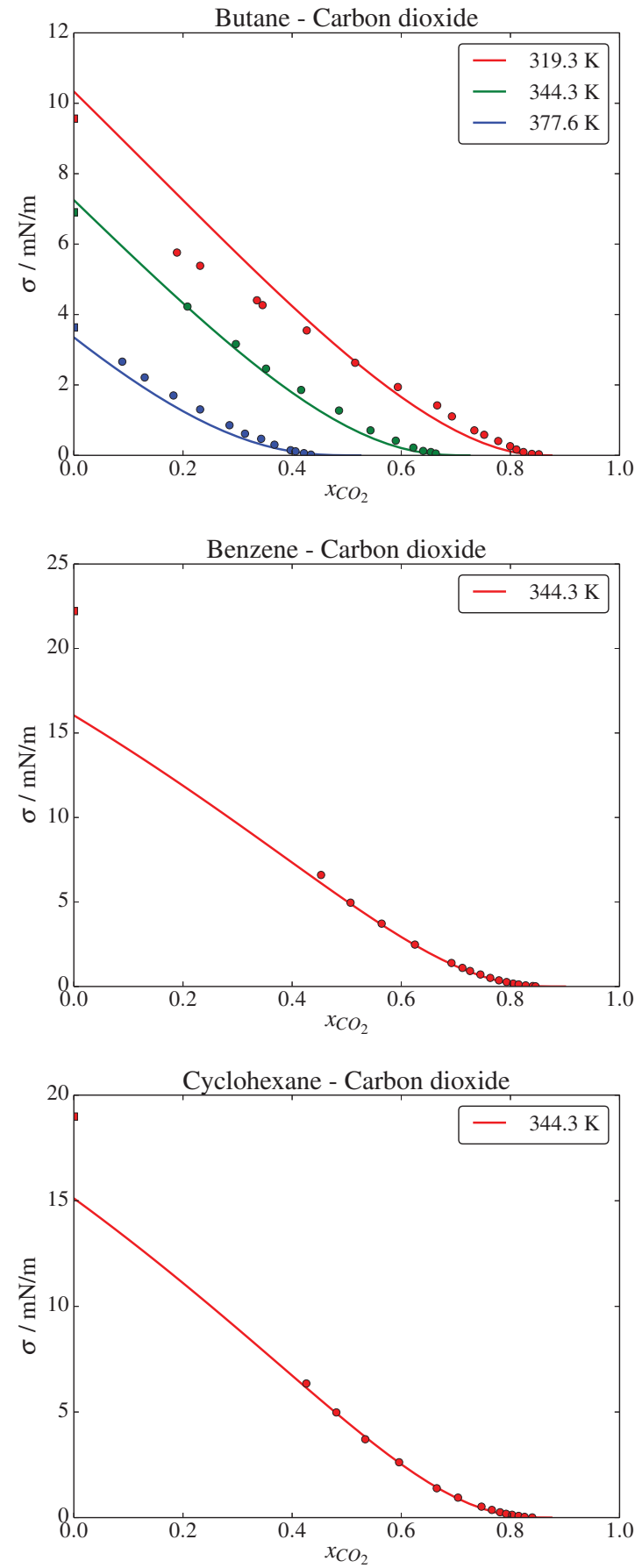

Fig. 1. Surface tension of binary mixtures predicted with the parachor parameters by Gasem et al. [10]. The experimental surface tension data $[12,13]$ are plotted as circles, and the pure solvent surface tension [19] is plotted with squares.

assuming a constant exponent $n=4$, and plotted as a function of the reduced temperature $T_{r}=T / T_{c}$, where $T_{c}$ is the critical temperature of the pure solvent. A common feature of all the parachors is that they are almost constant up to a reduced temperature of roughly 0.8 . Above this value, the parachors increase steeply, by more than $30 \%$ when approaching the critical point.

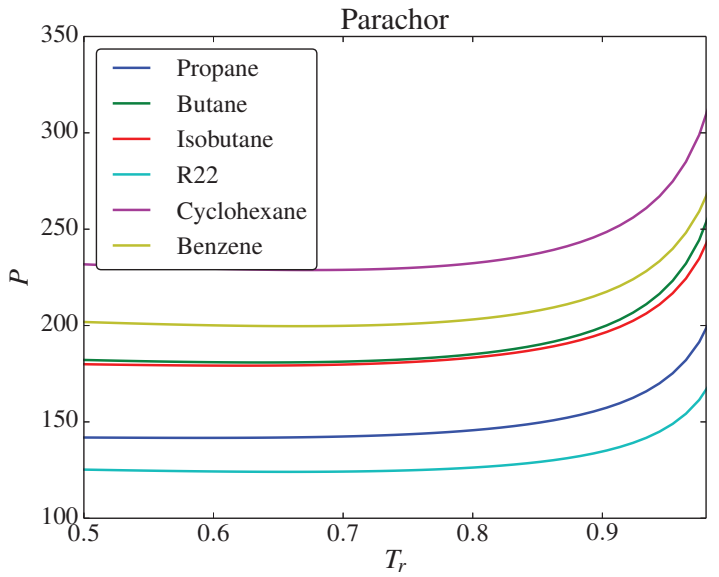

Fig. 2. Solvent parachors fitted with a constant exponent $n=4$ (case 1).

Figure 3 shows the fitted solvent parachors and the corresponding exponent $n$ for the case 2. The parachors feature a common functional dependence on $T_{r}$, similarly to the case 1 . However, the parachor is almost a linearly increasing function of the reduced temperature. The exponent $n$ is a linearly decreasing function of temperature; $n$ being around 4.5 at $T_{r}=0.5$, and around 2.5 when approaching the critical temperature of the solvent. Interestingly, $n$ is almost identical for all the binary mixtures considered in this analysis.

The predictions of the parachor method comparing the two scenarios of parachor parameters estimation, case 1 and case 2 , are shown in figure 4 . The predictions of case 1 are plotted as dashed lines, and the predictions of case 2 are drawn as full lines. For all the three mixtures, the predictions of case 2 show a better agreement with the binary experimental surface tension data, although for benzene and cyclohexane the improvement is almost negligible.

\subsection{Parachor method predictions for refrigerant mixtures}

The binary mixtures of a refrigerant and carbon dioxide were investigated extensively in bubble nucleation studies [15]. There is a wide range of experimental bubble nucleation data available in the literature [20]. However, the theoretical explanation of the measured nucleation rates was not given with sufficient precision yet. One of the reasons for the failure of the theoretical predictions of bubble nucleation rate in the binary case is the lack of knowledge of the surface tension of these mixtures. So far, the theoretical predictions utilized the pure-solvent surface tension [15], which is considerably higher than the surface tension of the mixture at higher concentrations of carbon dioxide. As the nucleating mixtures are typically rich in carbon dioxide, the approximation of the binary surface tension with the pure-solvent surface tension is rather crude.

The surface tension of three refrigerant - carbon dioxide mixtures, i.e. with propane, isobutane, and R22, respectively, is shown in figure 5 as predicted by the parachor 

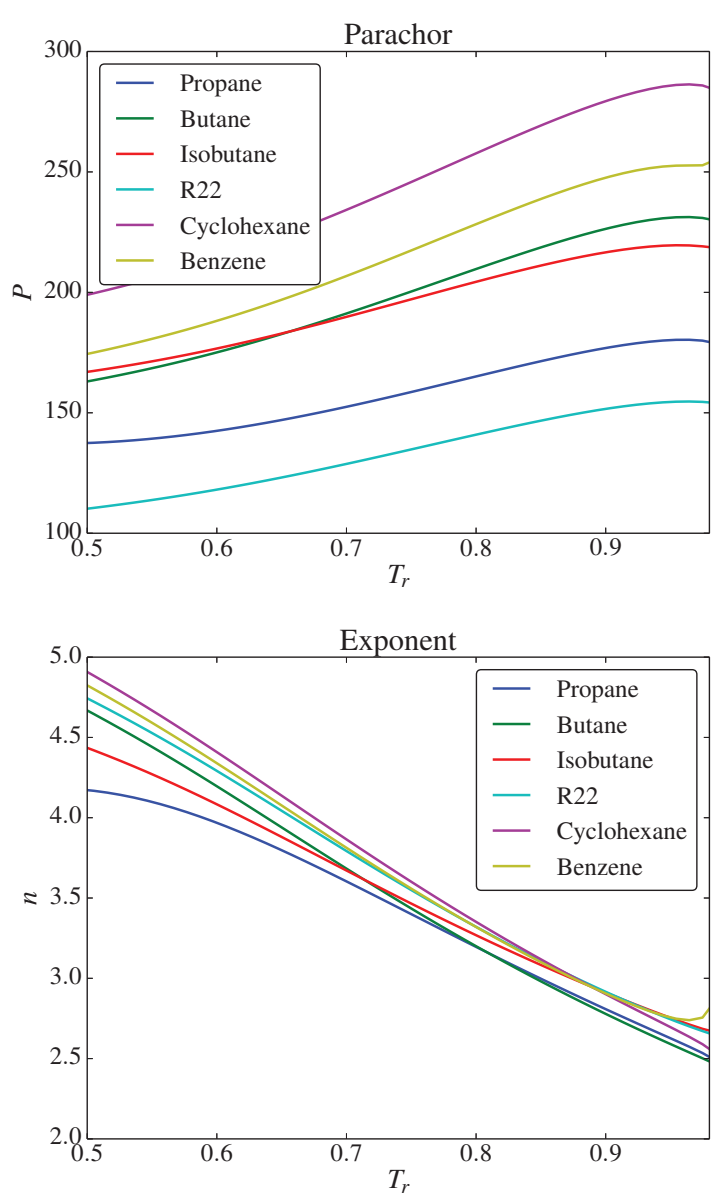

Fig. 3. Solvent parachors and exponent fitted with Eq. (1) to the surface tension data of pure solvents (case 2).

method. The case 2 version of parachor parameters fitting was used; the parachor parameters used in calculating the surface tension in figure 5 are those from figure 3.

\subsection{Parachor scaling}

Zhelezny et al. [6] found a correlation between the critical parachor $P_{c}$ and the molecular volume $v_{c}\left[\mathrm{~cm}^{3} / \mathrm{mol}\right]$. Their critical parachor is defined as $P_{c}=M \sigma_{0}^{\beta / \mu} / \varrho_{0}$, where $M$ is the molecular weight, $\sigma_{0}$ and $\varrho_{0}$ are the constants of the critical scaling of the surface tension $\sigma=\sigma_{0}\left(1-T_{r}\right)^{\mu}$ and of the VLE density difference $\varrho_{l}-\varrho_{g}=\varrho_{0}\left(1-T_{r}\right)^{\beta}$, respectively, and $\beta=1.260$ and $\mu=0.325$ are the critical exponents.

The relation, $P_{c}=0.75 v_{c}$, between the critical parachor and the critical volume was confirmed for a set of 26 substances (refrigerants, alkanes, atmospheric gases) by Zhelezny et al. To investigate the scaling behavior of the temperature-dependent parachors calculated in this work, the parachors from the case 2 (figure 3 ) are plotted in figure 6 and are scaled by the critical volume of the respective substance. The factor 0.75 found in the work of Zhelezny et al. was omitted in this scaling, because it is related to their specific choice of the critical parachor $P_{c}$, which uses
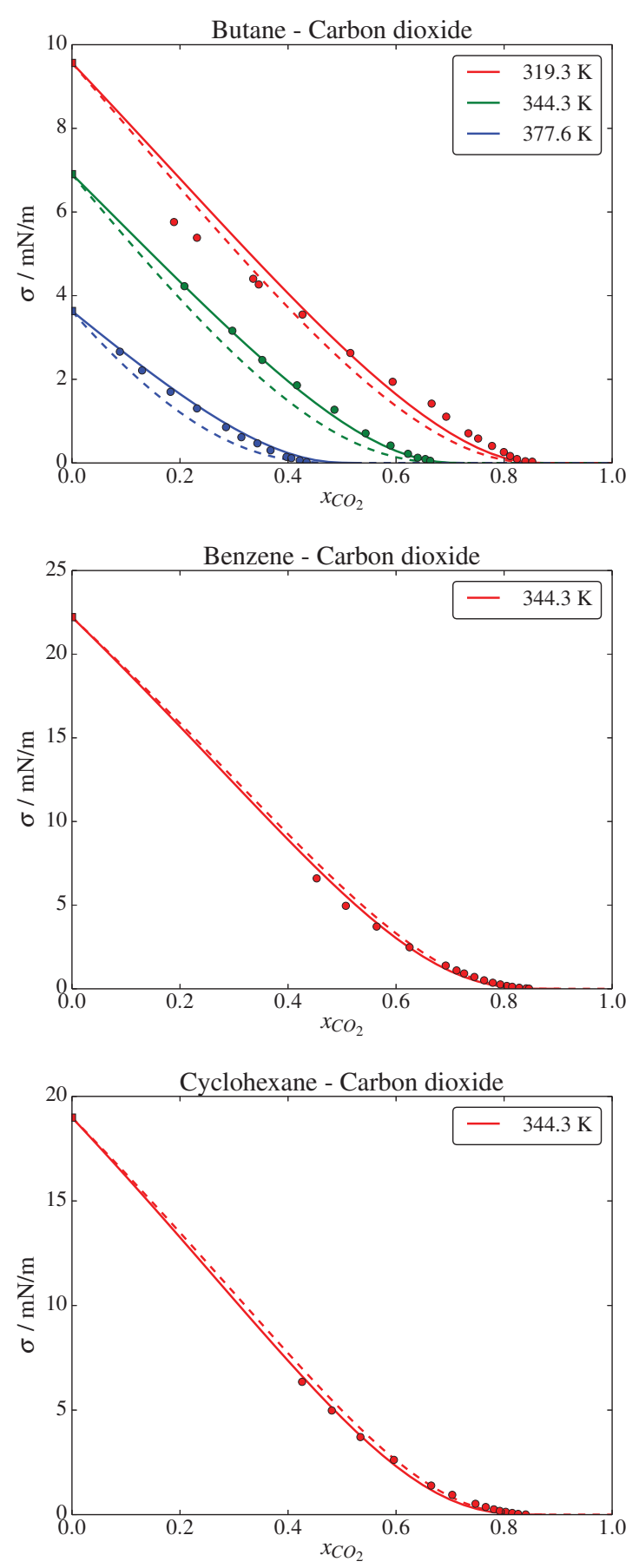

Fig. 4. Comparison of the surface tension of binary mixtures predicted by two methods of parachor parameter estimation, case 1 (dashed lines) and case 2 (full lines). The experimental surface tension data $[12,13]$ are plotted as circles, and the pure solvent surface tension [19] is plotted with squares. 

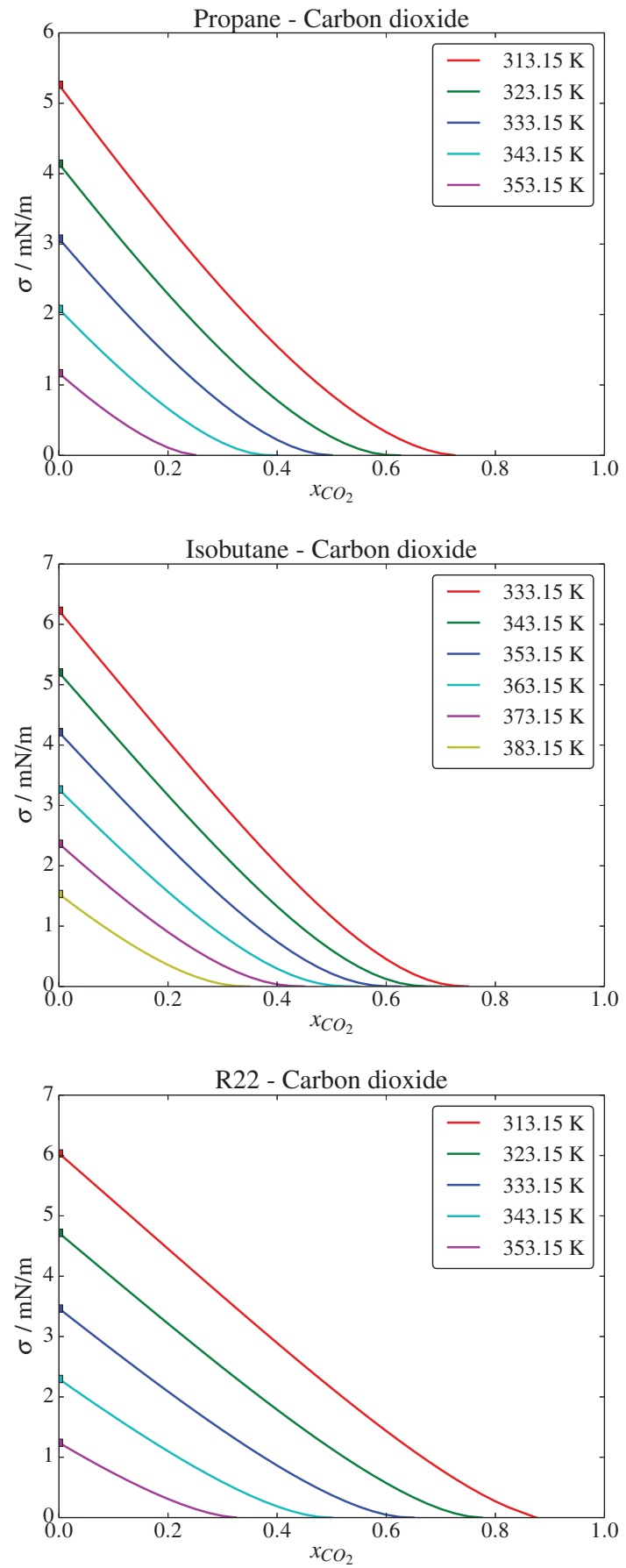

Fig. 5. Surface tension of refrigerant mixtures with carbon dioxide relevant to bubble nucleation studies [15] predicted by the parachor method with temperature-dependent parachor $P_{1}$ and exponent $n$ (case 2).

the exponent $n=\beta / \mu=3.87$. Since, in this analysis, the exponent $n$ is resulting from the fitting procedure, and its value reaches 2.5 close to the critical point, the factor 0.75 has no relevance here.

No definite statement, however, can be made about the scaled parachors in figure 6 . The dependence of the scaled parachor on the reduced temperature is almost identical for the 4 out of 6 substances, i.e. propane, butane, cyclohex-

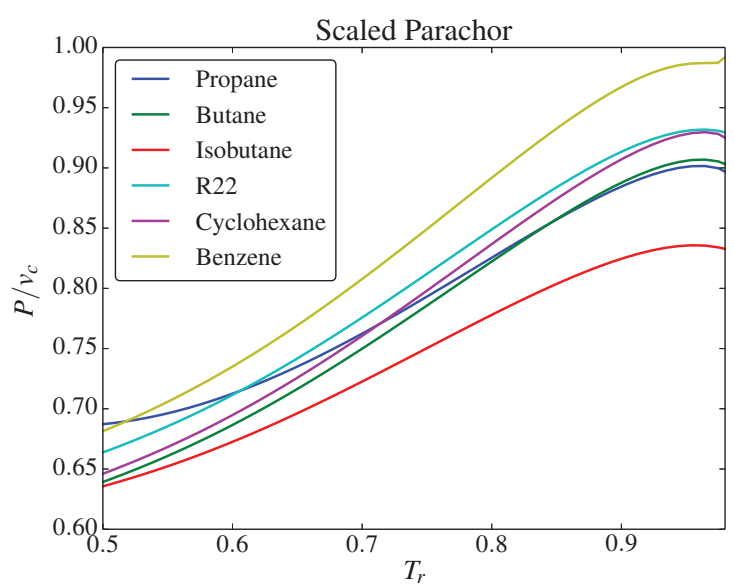

Fig. 6. Parachor values from case 2 scaled by the critical volume $v_{c}\left[\mathrm{~cm}^{3} / \mathrm{mol}\right]$.

ane, and R22. But the remaining two substances, i.e. benzene and isobutane, show a $10 \%$ deviation from the behavior of the other substances. Moreover, the scaled parachor of isobutane is lower, and the scaled parachor of benzene is higher than the scaled parachors of the other substances. The scaled parachors roughly coincide at low reduced temperatures only, e.g. at $T_{r}=0.5$ the scaled parachors $P / v_{c}$ equal $0.66 \pm 0.02$.

\section{Conclusion}

A modification of the parachor method for the prediction of the surface tension of binary mixtures of carbon dioxide was studied in this work by considering the possibility of temperature-dependent parameters of the parachor relation. For the case of binary mixtures of carbon dioxide, a procedure for the calculation of the temperature-dependent parachor parameters from the experimental pure-solvent surface tension data was presented, and a considerable improvement of the predictive capability of the parachor method was observed. Although the parachor parameters were fitted to the experimental surface tension of pure solvents only, the binary surface tension data from independent literature sources are estimated very accurately. The absence of the need for a binary mixing rule, or a binary interaction parameter, to accurately estimate the binary surface tension data makes this method quite robust.

A quite simple temperature dependence of the parachor parameters resulted from this analysis. The individual solvent parachors increase almost linearly with the reduced temperature. The exponent of the parachor equation decreases with the reduced temperature uniformly for all the binary mixtures investigated in this work.

\section{Acknowledgement}

I would like to acknowledge the institutional support RVO:61388998, the support by the Czech Science Foundation (projects GAP101/10/1819 and GA13-23550S), and 
by the Technology Agency of the Czech Republic (project TA03030978).

\section{References}

1. S.N. Balasubrahmanyam, Curr. Sci. India 94, 1650 (2008)

2. D. Macleod, Trans. Faraday Soc. 19, 0038 (1923)

3. S. Sugden, J. Chem. Soc. 125, 1177 (1924)

4. V.P. Zhelezny, E.G. Bysko, Int. J. Thermophys. 20, 1699 (1999)

5. D. Broseta, Y. Meleán, C. Miqueu, Fluid Phase Equilib. 233, 86 (2005)

6. V.P. Zhelezny, Y.V. Semenyuk, S.N. Ancherbak, N.V. Emel'yanenko, Russ. J. Phys. Ch. 83, 182 (2009)

7. H. Lin, Y.Y. Duan, Q. Min, Fluid Phase Equilib. 254, 75 (2007)

8. D. Fu, Y. Wei, Ind. Eng. Chem. Res. 47, 4490 (2008)

9. V. Vins, B. Plankova, J. Hruby, Int. J. Thermophys. 34, 792 (2013)

10. K. Gasem, P. Dulcamara, K. Dickson, R. Robinson, Fluid Phase Equilib. 53, 39 (1989), 5th International Conf On Fluid Properties And Phase Equilibria For Chemical Process Design, Banff Ctr, Banff, Canada, Apr 30 - May 05, 1989

11. Y.X. Zuo, E.H. Stenby, Can. J. Chem. Eng. 75, 1130 (1997)

12. J. Hsu, N. Nagarajan, R. Robinson, J. Chem. Eng. Data 30, 485 (1985)

13. N. Nagarajan, R. Robinson, J. Chem. Eng. Data 32, 369 (1987)

14. F.J. Blas, F.J. Martinez-Ruiz, A.I. MorenoVentas Bravo, L.G. MacDowell, J. Chem. Phys. 137 (2012)

15. Y. Mori, K. Hijikata, T. Nagatani, Int. J. Heat Mass Transfer 19, 1153 (1976)

16. S. Vitu, R. Privat, J.N. Jaubert, F. Mutelet, J. Supercrit. Fluid. 45, 1 (2008)

17. J. Vrabec, Y. Huang, H. Hasse, Fluid Phase Equilib. 279, 120 (2009)

18. J.M. Smith, H.C. Van Ness, Introduction to Chemical Engineering Thermodynamics, 4th edn. (McGrawHill Book Company, 1987)

19. A. Mulero, I. Cachadiña, M.I. Parra, J. Phys. Chem. Ref. Data 41, 043105 (2012)

20. C.T. Avedisian, J. Phys. Chem. Ref. Data 14, 695 (1985) 